\title{
Diastolic dysfunction in asymptomatic type 2 diabetes mellitus with normal systolic function
}

\author{
Virendra C. Patil, Harsha V. Patil'1, Kuldeep B. Shah, Jay D. Vasani, Pruthvi Shetty \\ Departments of Medicine, 'Microbiology, and Krishna Institute of Medical Sciences University (KIMSU), \\ Satara, Maharashtra, India \\ Address for correspondence: Dr. Virendra C. Patil, Department of Medicine, Krishna Institute of Medical \\ Sciences University (KIMSU), Dhebewadi Road, Karad Dist: Satara, Maharashtra - 415110, India \\ E-mail:virendracpkimsu@rediffmail.com
}

\begin{abstract}
Background: The incidence of heart failure in diabetic subjects is high even in the absence of hypertension and coronary artery disease. Aims: The purpose of this study was to study the incidence of diastolic dysfunction in diabetic subjects and its relation to age, duration of diabetes mellitus (DM), Glycosylated hemoglobin (HbA1c) levels, obesity indices and diabetic microangiopathies. Settings and Design: This was a case control prospective study conducted at the teaching hospital during a one year period. Materials and Methods: A total of 127 subjects (case) with type 2 diabetes of more than five years duration were studied. Total 100 healthy subjects were included as the control group. Echocardiography was performed to assess left ventricular diastolic function. Results: Out of the total 127 subjects, 69 (54.33\%) from the case group had diastolic dysfunction, and 11\% amongst 100 in the control group population showed the diastolic dysfunction $(P<0.001)$. Patients with a longer duration of DM (of 11 to 15 years) had a higher prevalence of diastolic dysfunction $(P<0.02)$. Subjects with high waist circumference and high waist to hip ratio had statistically significant diastolic dysfunction with ' $P$ ' $=0.001$ and ' $P$ ' $=<0.02$ respectively. Subjects with $\mathrm{HbA1c}>7.5 \%$ had a higher prevalence of diastolic dysfunction than subjects with $\mathrm{HbA} 1 \mathrm{c}<7.5 \%(P<0.02)$. Diastolic dysfunction was present in majority of the subjects with autonomic neuropathy and retinopathy. Conclusions: Present study reveals high incidence of diastolic dysfunction in asymptomatic diabetic; subjects and, this finding was correlated with the duration of diabetes, HbAlc levels, obesity indices and diabetic microangiopathies. We conclude that early diagnosis and institution of treatment will reduce morbidity and improve the outcomes, and prevent future heart failure.
\end{abstract}

Key words: Diastolic dysfunction, diabetes mellitus, echocardiography, heart failure

\section{INTRODUCTION}

The incidence of diabetes mellitus (DM) is increasing worldwide and rapidly assuming epidemic proportions. Over the last three decades, a number of epidemiological, clinical and autopsy studies have proposed the presence of

\begin{tabular}{|l|l|}
\hline \multicolumn{2}{|c|}{ Access this article online } \\
\hline Quick Response Code: & Website: \\
\hline & www.jcdronline.com \\
\cline { 2 - 2 } & \\
\hline
\end{tabular}

diabetic heart disease as a distinct clinical entity. Diastolic heart failure (HF) is also referred to as $\mathrm{HF}$, with preserved left ventricular systolic function. Many studies have reported that the incidence of heart failure in diabetic subjects is high even in the absence of hypertension and coronary artery disease. Studies have reported a high prevalence of pre-clinical diastolic dysfunction among subjects with DM. ${ }^{[1]}$ The evidence indicates that myocardial damage in diabetic subjects affects diastolic function before the systolic function. The pathogenesis of this left ventricular (LV) dysfunction in diabetic subjects is not clearly understood.

Diabetic cardiomyopathy has been proposed as an independent cardiovascular disease, and many mechanisms, 
such as microvascular disease, autonomic dysfunction, metabolic disorders, and interstitial fibrosis, have been suggested as causative factors. ${ }^{[2]}$ However, the exact etio-pathogenesis of diabetic cardiomyopathy still remains unclear. So far, very few population-based studies have been carried out in India, to demonstrate the prevalence of diastolic dysfunction in diabetic subjects in the Indian patients. The objective of our study was to determine whether there is any association between diastolic dysfunction and type 2DM, even in the asymptomatic subjects. Thus, this prospective case control study was conducted with the aim of determining the prevalence of asymptomatic LV diastolic dysfunction in type 2 diabetes subjects, and its relation to age, duration of DM, HbA1c, obesity indices and other diabetic complications such as microangiopathies.

\section{MATERIALS AND METHODS}

\section{The objectives of our study}

- To determine the incidence of LV diastolic dysfunction in asymptomatic type $2 \mathrm{DM}$ patients and to compare it with normal subjects;

- and, to quantify the relation of LV diastolic dysfunction with age, duration of DM, HbA1c, obesity indices and other diabetic complications such as microangiopathies.

For the study, we hypothesized that the diastolic dysfunction, [as assessed by the mitral peak velocity of early filling (E) to early diastolic mitral annular velocity (e') (E/e') ratio], worsens with age, duration of DM, HbA1c and obesity indices. A total of 127 normotensive subjects (case), with type $2 \mathrm{DM}$ of more than five years duration with no clinical evidence of cardiac disease were studied. A total 100 apparently healthy subjects with age and sex matched were included as the control group. This casecontrol study was designed to determine the prevalence of asymptomatic left ventricular diastolic dysfunction in type $2 \mathrm{DM}$ subjects and its relation to patient's age, duration of $\mathrm{DM}$, control of diabetes as determined by HbA1c levels, biochemical profile and obesity indices. This was a case -control prospective, observational study conducted out at the Krishna Institute of Medical Sciences, Karad, over a period of one year period from January 2009 to December 2009. This study was approved by the ethical committee of Krishna Institute of Medical Sciences University Karad.

\section{Inclusion criteria for case population}

All type $2 \mathrm{DM}$ with patients with duration $>5$ years with normal left ventricular systolic function (LVEF: $\geq 50 \%$ ).

\section{Exclusion criteria for case population}

- Subjects with evidence of coronary artery disease - CAD [excluded by history of angina, chest pain, Electrocardiogram (ECG) changes and abnormal Treadmill test (TMT) results];

- $\quad$ subjects with evidence of valvular disease;

- hypertensive patients, antihypertensive agents and/ or angiotensin-converting enzyme (ACE) inhibitors, with evidence of left ventricular hypertrophy on echocardiography;

- and, subjects with poor transthoracic echo window.

Detailed medical history was collected from each eligible subject; and, they underwent physical examination and biochemical investigations. After a 12 -hour fast, a venous blood sample was collected and sent to the biochemistry laboratory for estimation of the following: plasma glucose level; glycatedHbA1c; total serum cholesterol (TC); High-density lipoprotein (HDL) cholesterol; Low density lipoprotein (LDL) cholesterol; Very low density lipoprotein (VLDL); and, serum triglyceride levels (TG). ECG was done in all subjects. Physical examination included routine general examination, systemic examination and anthropometric evaluation including height (meter), hip circumference in centimetre (HC), waist circumference in centimetre (WC) and weight in kilogram.

\section{Echocardiography}

All the subjects were underwent resting transthoracic 2-dimensional echocardiography and Doppler imaging, to assess left ventricular diastolic function. Echocardiographer was not aware of this study to avoid bias in the interpretation. A transthoracic 2-dimentionsional echocardiogram (TTE) with pulsed Doppler evaluation of transmitral inflow and Tissue Doppler Imaging (TDI) and 2D echocardiography was performed to minimize the errors in assessing the diastolic dysfunction. Echocardiography was performed by harmonic imaging mode by Acuson-Siemens-X 300 echocardiography machine (5-1 $\mathrm{MHz}$ multi-frequency probe) according to the standard protocol. Pulsed-wave Doppler (PWD)-derived transmitral inflow velocities was obtained in the apical 4-chamber view, with the sample volume placed at the mitral valve leaflet tips. ${ }^{[3]}$ Measurements included the transmitral early diastolic rapid filling (E-wave) and atrial contraction late filling (A-wave) velocities to calculate $\mathrm{E} / \mathrm{A}$ ratio, isovolumteric relaxation time (IVRT) and deceleration time (DT). For tissue Doppler imaging, the mitral annulus velocity was obtained with a $2 \mathrm{~mm}$ sample volume placed at the 
lateral side and septal side of the mitral annulus. Diastolic dysfunction was labelled according to the standard guidelines. Left ventricular overall ejection fraction (systolic function) was calculated by modified Simpson's method; and, LVEF $\geq 50 \%$ was considered as normal. ${ }^{[3,4]}$ All echocardiographic measurements were averaged over three consecutive cardiac cycles, measured by a single investigator blinded to all other variables [Figure 1].

\section{Diagnostic criteria}

- Dyslipidemia: was defined if TC $\geq 200 \mathrm{mg} / \mathrm{dL}$; LDL cholesterol $\geq 130 \mathrm{mg} / \mathrm{dL}$; HDL cholesterol $40 \mathrm{mg} /$ $\mathrm{dL}$; and, $\mathrm{TG} \geq 150 \mathrm{mg} / \mathrm{dL}$. ${ }^{[5]}$

- Obesity indices: Cut-off for high Body Mass Index (BMI) was $\geq 25$ for females and $\geq 27$ for males. Cutoff for high waist to hip ratio (WHR) was $\geq 0.9$ for males, and $\geq 0.8$ for females. Cut-off for high WC was $>85 \mathrm{~cm}$ for females and $>90 \mathrm{~cm}$ for males. ${ }^{\left[{ }^{[6]}\right.}$

- Diabetes mellitus (DM): If a subject is a known diabetic on treatment, or with any fasting blood sugar level (F-BSL) $\geq 126 \mathrm{mg} / \mathrm{dL}^{\left[{ }^{[6]}\right.}$

- Retinopathy: Microangiopathy was assessed by fundoscopy (direct ophthalmoscopy). The ophthalmologist doing fundoscopy was unaware of this study. Fundoscopic examination was done after dilating the pupil with tropicamide (1\%). Retinopathy status was labeled as follow:

- no evidence of diabetic retinopathy;
- background diabetic retinopathy, defined as presence of one or more microaneurysms, punctate or striate intraretinal hemorrhages, and hard exudates;

- preproliferative diabetic retinopathy defined as soft exudates, venous beading, and intraretinal microvascular abnormalities;

- proliferative diabetic retinopathy characterized by neovascularization on or within one disk diameter of the disk in extent. After these initial evaluations were completed, 127 diabetic subjects were enrolled into the study protocol. Hundred apparently healthy individuals, matched for age and sex served as control group. ${ }^{[7]}$

- Autonomic neuropathy: Autonomic function was evaluated by unmasking the sympathetic dysfunction by the blood pressure (BP) response to standing. A fall in systolic BP on erect position of $<10 \mathrm{mmHg}$ was defined as normal and $>30$ $\mathrm{mmHg}$ as abnormal. ${ }^{[7]}$

- Diastolic dysfunction: LV diastolic dysfunction was considered to be present if any of the following findings were seen, as previously described: ${ }^{[3,4]}$

- $\mathrm{E} / \mathrm{A}$ ratio $<1$ or $>2$

- $\mathrm{DT}<150$ or $>220 \mathrm{~ms}$,

- IVRT $<60$ or $>100 \mathrm{~ms}$, or

- E/e' ratio $>15$

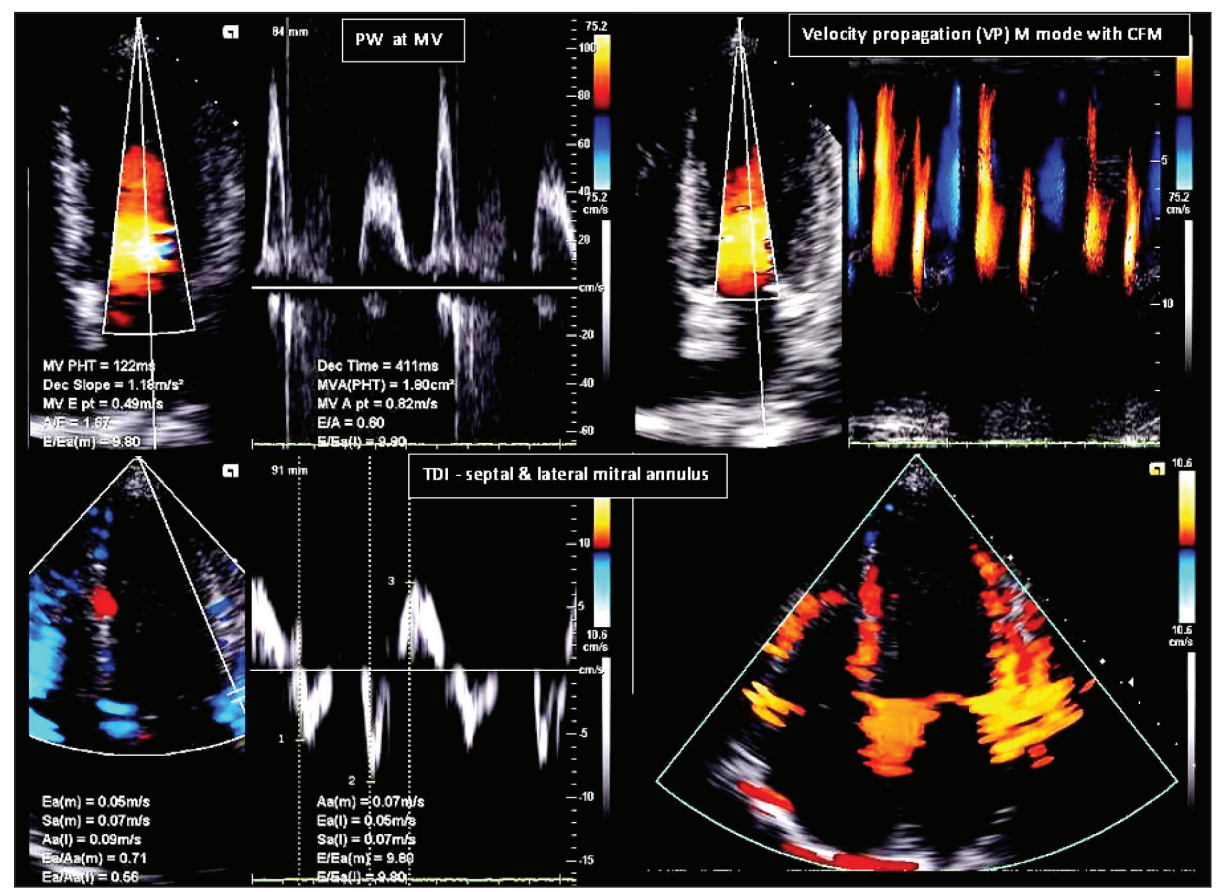

Figure 1: Two dimensional transthoracic echocardiographic evaluation of diastolic dysfunction by pulse wave Doppler (PW) at mitral valve, velocity propagation (VP) by colour ' $\mathrm{M}$ ' mode and tissue Doppler imaging (TDI) at septal and lateral mitral annulus 


\section{Statistical analysis}

Data was analysed for mean, percentage, standard deviation, chi square test, multiple correlation and multivariate analysis, by using SPSS-10 (Statistical Package for the Social Sciences) for Windows (SPSS, Chicago, IL). Variables that were not normally distributed were reciprocally transformed for analysis. The 't'-test and Chi-Square tests were applied to study quantitative and qualitative data, respectively with ' $P$ ' value $<0.05$ was considered statistically significant. Correlation of various factors was determined using $r^{2}$ and multiple linear regression analysis. Correlation $(r)$ findings were describes as follows: $r=0.8$ (high correlation coefficient); $r=0.4-0.7$ (moderate correlation); and, $r=0.3$ and above (low correlation coefficient).

\section{RESULTS}

A total 127 subjects with type-2 diabetes mellitus (cases) and 100 healthy age and sex matched controls were included in this case-control prospective study. Out of 127 subjects with type -2 DM, $69(54.93 \%)$ were male and $58(46.66 \%)$ were female. Total $56(54 \%)$ male and $44(46 \%)$ female were control healthy subjects. Mean standard deviation of biochemical, anthropometric indices and echocardiographic parameters were obtained [Table 1]. Mean of BMI in the case group was significantly higher as compared to the control group (' $P$ ' $<0.05)$. Mean of WC in the case group was significantly higher as compared to the control group $\left({ }^{\prime} P^{\prime}=0.02\right)$. Mean of WHR in the case group was significantly higher as compared to the control group (' $P$ ' $<0.02)$. Mean of TC in case group was high compared to control group. Mean of TG in the case group was significantly higher as compared to control group (' $P$ ' $=0.032$ ). Mean of LDL- cholesterol in the case group was higher as compared to the control group. Mean of HDL- cholesterol in the case group was significantly lower as compared to the control group (' $P$ ' $<0.05)$. Mean of BSL in the case group was significantly higher as compared to the control group (' $P$ ' $=0.01$ ). Mean of $\mathrm{E} / \mathrm{A}$ ratio in the case group was significantly lower as compared to the control group (' $P$ ' $=0.02$ ). Mean of $\mathrm{E} / \mathrm{e}$ ' ratio in the case group was significantly higher as compared to the control group ( $P$ ' $<0.001$ ). Total $69(54.33 \%)$ subjects from the case group had diastolic dysfunction; and, 11 (11\%) amongst control group had the diastolic dysfunction. Diastolic dysfunction in type -2 DM subjects was significantly higher as compared to the control group ( $P$ ' $<0.001$ ) with odds ratio of 2.18 [Table 1].

\section{Relation of diastolic dysfunction with various dependent variables in type 2 diabetes subjects}

Out of 89 subjects with HbA1c < 7.5\%, 39 (42.82\%) had diastolic dysfunction; and, out of 38 subjects with HbA1c $>7.5 \%, 31(81.57 \%)$ had diastolic dysfunction. Subjects with HBA1c $>7.5 \%$ had more prevalence of

Table 1: Mean and standard deviation of numerical parameters of the patients in the study

\begin{tabular}{|c|c|c|c|c|c|}
\hline \multirow[t]{2}{*}{ Variables } & \multicolumn{2}{|c|}{ Case population } & \multicolumn{2}{|c|}{ Control population } & \multirow[t]{2}{*}{ ' $P$ ' value } \\
\hline & $\begin{array}{c}\text { Male } \\
(n=69)\end{array}$ & $\begin{array}{c}\text { Female } \\
(n=58)\end{array}$ & $\begin{array}{c}\text { Male } \\
(n=56)\end{array}$ & $\begin{array}{c}\text { Female } \\
(n=44)\end{array}$ & \\
\hline Age (years) & $51 \pm 9$ & $49 \pm 10$ & $\pm 49 \pm 7$ & $48 \pm 8$ & NS \\
\hline Duration of diabetes (years) & $11 \pm 5$ & $10 \pm 4$ & - & - & - \\
\hline Body mass index $\left(\mathrm{Kg} / \mathrm{m}^{2}\right)$ & $27.6 \pm 2.2$ & $26 \pm 2.5$ & $23.5 \pm 1.6$ & $23.7 \pm 1.3$ & $<0.05$ \\
\hline Waist circumference (WC - cm) & $92 \pm 8$ & $78 \pm 7$ & $77 \pm 3.9$ & $74 \pm 6$ & 0.02 \\
\hline Waist to hip ratio (WHR) & $0.92 \pm 0.17$ & $0.84 \pm 0.19$ & $0.75 \pm 0.19$ & $0.75 \pm 0.15$ & $<0.02$ \\
\hline Total cholesterol & $213 \pm 24.4$ & $223 \pm 25$ & $147 \pm 8.7$ & $135 \pm 15$ & NS \\
\hline Triglyceride & $208 \pm 25.7$ & $198 \pm 23.5$ & $135 \pm 7.9$ & $129 \pm 9$ & 0.032 \\
\hline LDL- cholesterol & $134 \pm 13$ & $145 \pm 10$ & $96 \pm 13$ & $106 \pm 7.8$ & NS \\
\hline HDL- cholesterol & $39 \pm 16$ & $38.5 \pm 13$ & $49.5 \pm 7.8$ & $43 \pm 5.7$ & $<0.05$ \\
\hline Blood sugar level & $145 \pm 16.7$ & $139 \pm 23.5$ & $85 \pm 7.8$ & $85 \pm 5.0$ & 0.01 \\
\hline $\mathrm{HbA} 1 \mathrm{c}(\%)$ & $8.3 \pm 1.91$ & $8.1 \pm 1.3$ & - & - & - \\
\hline E/A ratio $(\mathrm{PW})$ & $0.79 \pm 0.13$ & $0.81 \pm 0.11$ & $1.21 \pm 0.22$ & $1.19 \pm 0.11$ & 0.02 \\
\hline E/e' ratio (TDI) & $18.6 \pm 3.5$ & $18.3 \pm 3.2$ & $8.8 \pm 1.24$ & $8.67 \pm 2.16$ & $<0.001$ \\
\hline IVRT (ms) & $79 \pm 9$ & $81 \pm 7$ & $95 \pm 14.8$ & $97 \pm 16$ & NS \\
\hline DT (ms) & $168 \pm 23$ & $172 \pm 19$ & $159 \pm 23.8$ & $139 \pm 11$ & $<0.002$ \\
\hline $\mathrm{EF}(\%)$ & $54 \pm 3$ & $55 \pm 2$ & $62 \pm 3$ & $49 \pm 3$ & NS \\
\hline Diastolic dysfunction & $39(56.52 \%)$ & $30(51.72 \%)$ & $6(10.71 \%)$ & $5(11.36 \%)$ & $<0.001$ \\
\hline
\end{tabular}




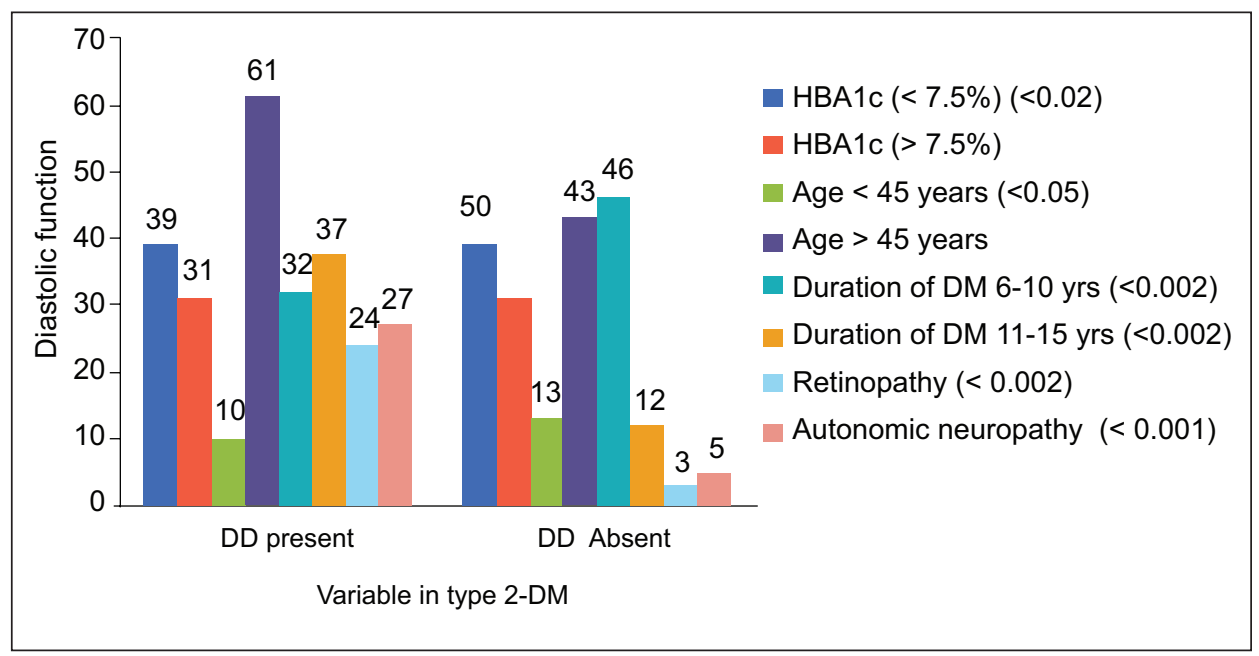

Figure 2: Relation of diastolic dysfunction with various dependent variables in type 2 DM

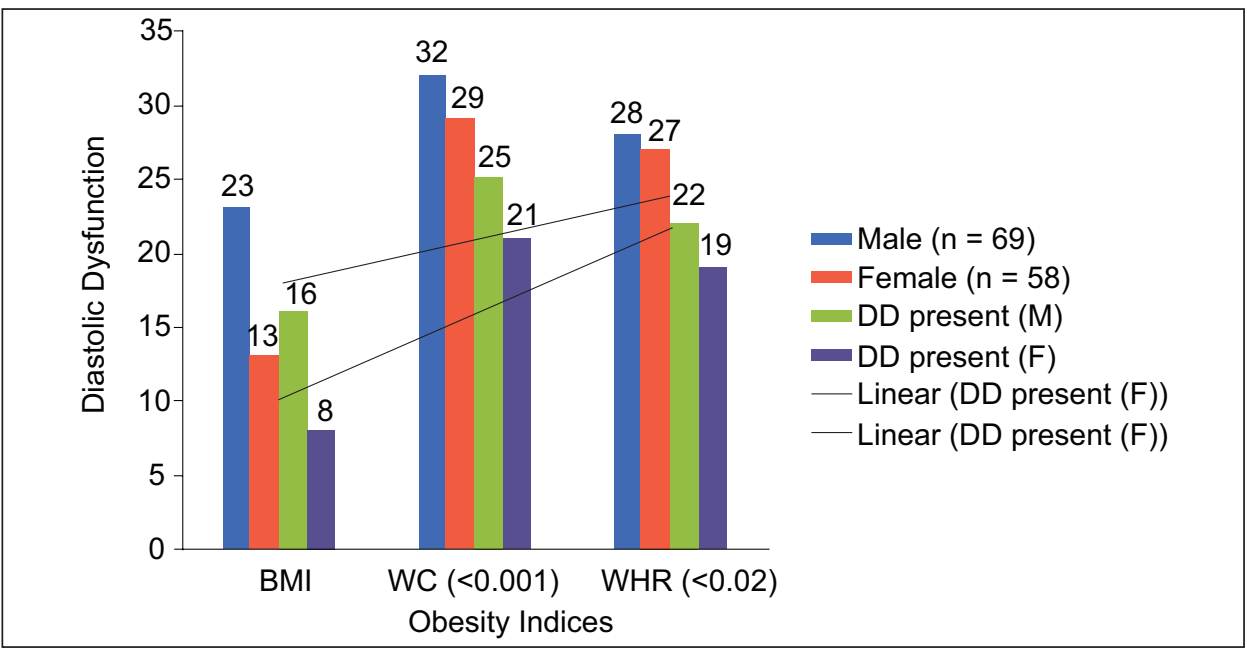

Figure 3: Relation of diastolic dysfunction with obesity indices in type 2 DM

diastolic dysfunction, than subjects with HBA1c $<7.5 \%$ (' $P$ ' $<0.02)$. Out of 23 subjects with age $<45$ years, $10(43.47 \%)$ had diastolic dysfunction; and, out of 104 subjects with age $>45$ years, $61(58.65 \%)$ had diastolic dysfunction. Diastolic dysfunction was significantly high in patients with age $>45$ years, compared to age $<45$ years ( $P$ ' $<0.05)$. Total $78(61.41 \%)$ subjects were with the duration of diabetes between 6-10 years, and 49 (38.58\%) were between $11-15$ years. Out of $78(61.41 \%)$ subjects with duration of diabetes between 6-10 years, 32 $(41.02 \%)$ had diastolic dysfunction. Out of 49 (38.58\%) subjects with duration of diabetes between 11-15 years, 37 (75.51\%) had diastolic dysfunction. Comparing duration of diabetes of 6 to 10 years and 11 to 15 years with diastolic dysfunction, patients with 11 to 15 years duration of diabetes had more prevalence of diastolic dysfunction $\left({ }^{\prime} P>0.02\right)$. Total $27(21.25 \%)$ subjects had retinopathy, of which $24(88.88 \%)$ had diastolic dysfunction ( $\left.{ }^{\prime} P<0.002\right)$.
Out of $32(25.19 \%)$ subjects with postural hypotension, $27(84.37 \%)$ had diastolic dysfunction ( $P$ ' $=0.001$ ) [Table 2 and Figure 2].

\section{Relation of diastolic dysfunction with obesity indices}

Total $23(33.33 \%)$ male and $13(24.41 \%)$ female patients had high BMI; and, out of them $16(69.56 \%)$ male and $8(61.53 \%)$ female had diastolic dysfunction. Total 32 (46.37\%) male and 29 (50\%) female patients had high WC; and, out of them, $25(78.12 \%)$ male and $21(72.41 \%)$ female patients had diastolic dysfunction (' $P$ ' $=0.001$ ). Total 28 (40.57\%) male and 27 (46.55\%) female patients had high WHR; and, out of them, 22 (78.57\%) male and $19(81.48 \%)$ female had diastolic dysfunction ('P' $=<0.02$ ). Subjects with high WC and high WHR had statistically significant diastolic dysfunction [Table 3 and Figure 3]. 
Patil, et al.: Diastolic dysfunction in type 2 diabetes mellitus with normal LVEF

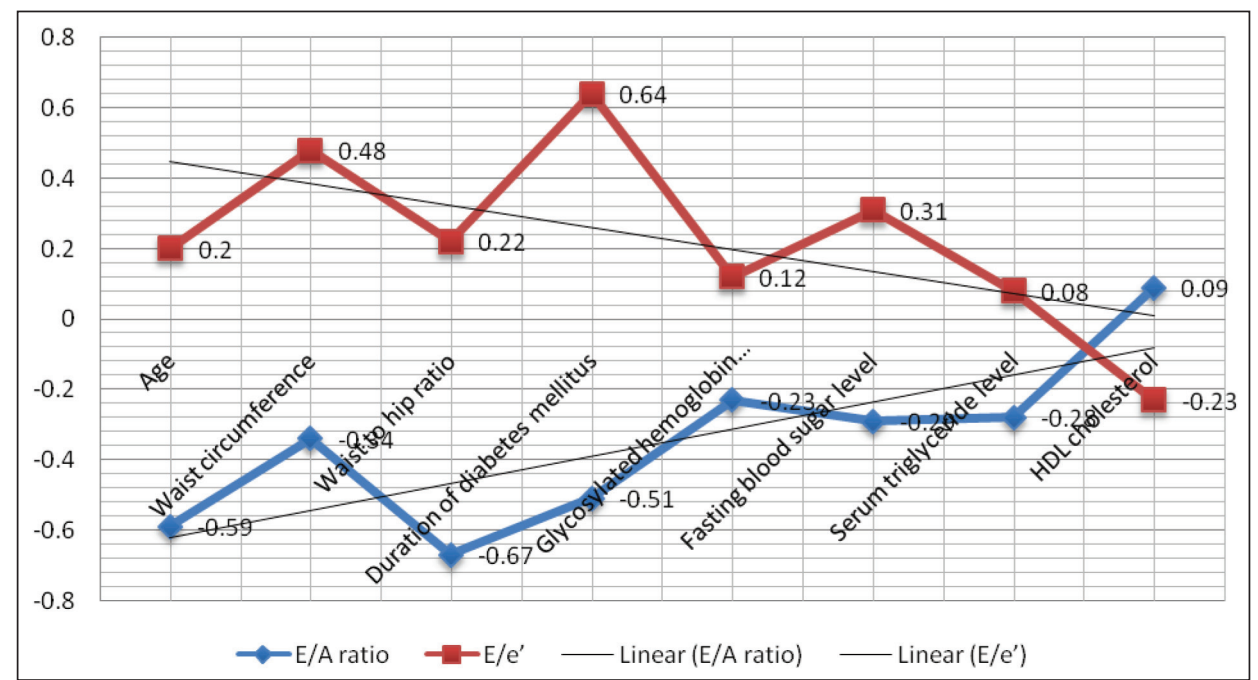

Figure 4: Correlation of parameters of diastolic dysfunction with biochemical profile and obesity indices in type 2 DM

Table 2: Relation of diastolic dysfunction with various dependent variables in type 2 diabetes mellitus subjects in the study

\begin{tabular}{|c|c|c|c|c|}
\hline Variables & $\begin{array}{c}\text { Diastolic dysfunction } \\
\text { present }\end{array}$ & $\begin{array}{c}\text { Diastolic dysfunction } \\
\text { absent }\end{array}$ & Total (\%) & ' $P$ ' value \\
\hline HBA1c $(<7.5 \%)(n=89)$ & $39(42.82)$ & 50 & 70.07 & $<0.02$ \\
\hline HBA1c $(>7.5 \%)(n=38)$ & $31(81.57)$ & 7 & 29.92 & \\
\hline Age $<45$ years $(n=23)$ & $10(43.47)$ & 13 & 18.11 & $<0.05$ \\
\hline Age $>45$ years $(n=104)$ & $61(58.65)$ & 43 & 81.88 & \\
\hline $\begin{array}{l}\text { Duration of diabetes mellitus } \\
6-10 \text { years }(n=78)\end{array}$ & $32(41.02)$ & 46 & 61.41 & $<0.002$ \\
\hline $\begin{array}{l}\text { Duration of diabetes mellitus } \\
11-15 \text { years }(n=49)\end{array}$ & $37(75.51)$ & 12 & 38.58 & \\
\hline Retinopathy present $(n=27)$ & $24(88.88)$ & 3 & 21.25 & $<0.002$ \\
\hline $\begin{array}{l}\text { Autonomic neuropathy (postural } \\
\text { hypotension) }(\mathrm{n}=32)\end{array}$ & $27(84.37)$ & 5 & 25.19 & $<0.001$ \\
\hline
\end{tabular}

Where, HbAlc $=$ glycosylated haemoglobin, Figures given in parenthesis are in percentage

Table 3: Relation of diastolic dysfunction with obesity indices of the patients in the study

\begin{tabular}{|c|c|c|c|c|c|}
\hline \multirow[t]{2}{*}{ Obesity indices } & \multirow[t]{2}{*}{ Male $(n=69)$} & \multirow[t]{2}{*}{ Female $(n=58)$} & \multicolumn{2}{|c|}{ Diastolic dysfunction present } & \multirow[t]{2}{*}{ ' $P$ ' value } \\
\hline & & & Male & Female & \\
\hline Body mass index $\left(\mathrm{Kg} / \mathrm{m}^{2}\right)$ & $23(33.33)$ & $13(24.41)$ & $16(69.56)$ & $8(61.53)$ & NS \\
\hline Waist circumference $(\mathrm{cm})$ & $32(46.37)$ & $29(50)$ & $25(78.12)$ & $21(72.41)$ & $<0.001$ \\
\hline Waist to hip ratio & $28(40.57)$ & $27(46.55)$ & $22(78.57)$ & $19(81.48)$ & $<0.02$ \\
\hline
\end{tabular}

$N S=$ not significant, Figures given in parenthesis are in percentage

Correlation of echocardiography parameters of diastolic dysfunction with obesity indices, duration of diabetes mellitus and biochemical profile

Age was negatively correlated with E/A ratio (-0.59); and, positively with $\mathrm{E} / \mathrm{e}$ ' ratio $(+0.2)$. WC was negatively correlated with $\mathrm{E} / \mathrm{A}$ ratio $(-0.34)$; and, positively with $\mathrm{E} / \mathrm{e}$ ' ratio $(+0.48)$. WHR was negatively correlated with $\mathrm{E} / \mathrm{A}$ ratio (-0.67); and, positively with $\mathrm{E} / \mathrm{e}^{\prime}$ ratio $(+0.22)$. Duration of diabetes was negatively correlated with $\mathrm{E} / \mathrm{A}$ ratio (-0.51); and, positively with $\mathrm{E} / \mathrm{e}^{\prime}$ ratio $(+0.64)$. GlycatedHbA1c level was negatively correlated with E/A ratio (-0.23); and, positively with $\mathrm{E} / \mathrm{e}^{\prime}$ ratio $(+0.12)$. Fasting BSL was negatively correlated with E/A ratio (-0.29); and, positively with $\mathrm{E} / \mathrm{e}^{\prime}$ ratio $(+0.31)$. Serum $\mathrm{TG}$ level was negatively correlated with E/A ratio (-0.28); and, positively with $\mathrm{E} / \mathrm{e}^{\prime}$ ratio $(+0.08)$. Serum $\mathrm{HDL}$ cholesterol levels were negatively correlated with $\mathrm{E} / \mathrm{e}$ ' ratio $(-0.23)$; and, positively with E/A ratio (+0.09) [Table 4 and Figure 4]. By multivariate analysis of covariance (MACOVA) and Cox's proportional hazard regression analysis, (after adjustment for age, sex) it was determined that the diastolic dysfunction (measured by E/A ratio and E/e' ratio), was significantly associated with longer duration of DM, HbA1c, serum 
Table 4: Correlation of parameters of diastolic dysfunction with biochemical profile and obesity indices of the patients in the study

\begin{tabular}{|c|c|c|}
\hline \multirow[t]{2}{*}{ Variables } & \multicolumn{2}{|c|}{$\begin{array}{c}\text { Echocardiographic } \\
\text { parameters of diastolic } \\
\text { dysfunction }\end{array}$} \\
\hline & E/A ratio & E/e' \\
\hline Age & -0.59 & +0.20 \\
\hline Waist circumference & -0.34 & +0.48 \\
\hline Waist to hip ratio & -0.67 & +0.22 \\
\hline Duration of diabetes mellitus & -0.51 & +0.64 \\
\hline Glycosylated hemoglobin (HbA1c) & -0.23 & +0.12 \\
\hline Fasting blood sugar level & -0.29 & +0.31 \\
\hline Serum triglyceride level & -0.28 & +0.08 \\
\hline HDL cholesterol & +0.09 & -0.23 \\
\hline
\end{tabular}

TG levels, WC and WHR, retinopathy and autonomic neuropathy (' $P$ ' $<0.002)$.

\section{DISCUSSION}

Our current findings demonstrate that pre-clinical diastolic dysfunction is common in patients with DM. Pre-clinical diastolic dysfunction has been broadly defined as diastolic dysfunction in patients with normal systolic function, and no symptoms of heart failure (HF). Present study reveals high burden of diastolic dysfunction in cohort of type 2 DM population. In the present prospective case control study, 127 subjects with type-2 DM as cases; and, 100 healthy subjects as controls, were included. Overall mean of obesity indices like BMI, WC and WHR were significantly higher in subjects with type $2 \mathrm{DM}$ compared to the control group. Mean of fasting BSL, HbA1c, serum TC, serum TG and LDL cholesterol in case group was significantly higher as compared to the control group. The mean of HDL cholesterol was lower in the case group as compared to the control group. Total $69(54.33 \%)$ subjects from the case group had diastolic dysfunction, and 11 (11\%) amongst control group showed the diastolic dysfunction. Diastolic dysfunction in type -2 diabetes subjects was significantly higher as compared to the control group ( $P$ ' $<0.001)$. E/A ratio negatively correlated with age, WC, WHR, duration of DM, HbA1c level, fasting BSL, and serum TG levels; and, positively correlated with serum HDL- cholesterol. E/e' ratio positively correlated with age, WC, WHR, duration of DM, HbA1c level, fasting BSL, and serum TG levels; and, negatively correlated with serum HDL- cholesterol. Duration of diabetes mellitus of 11 to 15 years had more prevalence of diastolic dysfunction (' $P$ ' $<0.02)$. Subjects with high WC and high WHR had statistically significant diastolic dysfunction. Subjects with HBA1c $>7.5 \%$ had more prevalence of diastolic dysfunction than subjects with HBA1c $<7.5 \%$ (' $P^{p}<0.02$ ). Diastolic dysfunction was significantly high in patient with age $>45$ years compared to age $<45$ years (' $P$ ' $<0.05)$. Diastolic dysfunction was present in majority of the subjects with autonomic neuropathy and retinopathy [Figure 2].

We compared our results with various studies. Soldatos et al. ${ }^{[8]}$ in their case control study of 55 individuals with type -2 DM found that Diastolic dysfunction, present in a significant proportion of population with Type $2 \mathrm{DM}$. Similarly, in the present study, $54.33 \%$ of subjects from the case group had diastolic dysfunction and $11(11 \%)$ amongst control group had the diastolic dysfunction $(P<$ 0.001). Sacre et al. ${ }^{[0]}$ found that there was an independent association between global cardiac autonomic neuropathy (CAN) and left ventricular (LV) dysfunction in patients with type $2 \mathrm{DM}$. These findings are comparable to our study, where diastolic dysfunction was present in majority of the subjects with autonomic neuropathy documented by postural hypotension. Out of the $32(25.19 \%)$ subjects with postural hypotension; 27 (84.37\%) had diastolic dysfunction (' $P=0.001)$. Van Heerebeek et al. ${ }^{[10]}$ in their study of 36 type $-2 \mathrm{DM}$ patients stated that, the cardiomyocyte resting tension is more important when LVEF is normal. Excessive diastolic left ventricular stiffness is an important contributor to heart failure in subjects with DM. Diabetes is presumed to increase stiffness through myocardial deposition of collagen and advanced glycation end products. Similarly, in the present study, $54.33 \%$ of subjects from the case group had diastolic dysfunction with normal LVEF.

Masugata et al..$^{[1]]}$ in their case control study of 77 normotensive patients found that, the cardiac diastolic dysfunction without LV systolic dysfunction in patients with well-controlled type $2 \mathrm{DM}$ is related neither to hypertension nor LV hypertrophy, but rather to aging and the duration of type 2 DM. Similarly, in the present study, total $54.33 \%$ of subjects from case group without hypertension and CAD had diastolic dysfunction with normal LV systolic function. Annonu et al. ${ }^{[12]}$ in their case control study of 66 subjects found that there was an inverse correlation between the duration of diabetes and $\mathrm{E} / \mathrm{A}$ ratio $(\mathrm{r}=-0.4$, ' $P$ ' $<.005)$. E/A ratio $<1$ was associated with a higher prevalence of retinopathy $(49 \%$ versus $20 \%$, ' $P$ ' $=0.01)$ and abnormal blood pressure response to standing $(29 \%$ versus $4 \%$, ' $P$ ' <.005). LV systolic and diastolic abnormalities are correlated with the duration of diabetes and with other diabetic microangiopathies, such as diabetic retinopathy and neuropathy. These results are comparable to the present study, where diastolic dysfunction was present in majority of the subjects with autonomic neuropathy and retinopathy with ' $P$ ' $=0.001$ and 
0.002 , respectively. Duration of diabetes mellitus of 11 to 15 years had more prevalence of diastolic dysfunction as compared to the $6-10$ years group $(' P$ ' $<0.02)$.

Mishra et al. ${ }^{[7]}$ in their case control study of 71 subjects with type $2 \mathrm{DM}$ found that asymptomatic diabetic patients have reduced LV systolic and diastolic function as compared with healthy subjects. LV systolic and diastolic abnormalities are correlated with the duration of diabetes and with diabetic microangiopathies, like retinopathy and neuropathy. These results are comparable with present study, where $54.33 \%$ of type -2 DM population had diastolic dysfunction and the DM was correlated to advancing age, increasing duration of DM, postural hypotension, retinopathy, high obesity indices, HbA1c $>7.5 \%$ and dyslipidemia.

From et al. ${ }^{[2]}$ in their study of 484 subjects between 1996 to 2007 year found that a duration of diabetes $\geq 4$ years was independently associated with LV diastolic dysfunction $\left(\mathrm{E} / \mathrm{e}^{\prime}>15\right)$ with odds ratio 1.91. Doppler imaging velocity of the medial mitral annulus during passive filling (E/e') ratio in diabetic patients is associated with the subsequent development of $\mathrm{HF}$ and increased mortality. Similarly in our study, duration of diabetes 1115 yrs had more prevalence of diastolic dysfunction as compared to the 6-10 year group (' $P$ ' $<0.02$ ). Sohail et al. ${ }^{[13]}$ in their study of 212 diabetic population found that $30.76 \%$ patients with type-2 DM had diastolic dysfunction. The LV diastolic dysfunction is much more prevalent in patients with type- 2 diabetes mellitus and LV diastolic dysfunction is an early marker of diabetic cardiomyopathy. In our study, prevalence of type $2 \mathrm{DM}$ was $54.33 \%$. Exiara et al. ${ }^{[14]}$ in their study of 114 subjects stated that the prevalence of LV diastolic dysfunction in normotensive, asymptomatic and well-controlled DM type 2 patients is high, and increases with age. A total of $63.2 \%$ patients had diastolic dysfunction in their study compared to our prevalence of $54.33 \%$. Diamant et al. ${ }^{[15]}$ stated that early (E) acceleration peak, deceleration peak, peak filling rate, and $\mathrm{E} / \mathrm{A}$ ratio, and all other indices of diastolic function, were significantly decreased in patients with recently diagnosed, well-controlled and uncomplicated type 2 diabetes compared with the controls (' $P$ ' $<0.02)$. These findings are similar to our results. Bonito, et al. ${ }^{[16]}$ stated that, an impairment of LV diastolic function occurs early in the natural history of type-2 DM, and is related to clinical evidence of microangiopathic complications. Aaron et al..$^{[17]}$ in 1,760 diabetic patients found that, 411 (23\%) patients had diastolic dysfunction and diabetic patients with diastolic dysfunction had a significantly higher mortality rate compared with those without diastolic dysfunction. An increase in the TDI velocity of the medial mitral annulus during passive filling (E/e') ratio in diabetic patients is associated with the subsequent development of HF. These findings are comparable with our study.

Boyer et al. ${ }^{[18]}$ stated that the prevalence of LV diastolic dysfunction in asymptomatic, normotensive patients with type 2 diabetes disease is high. Diastolic dysfunction was found in $75 \%$ subjects. They also found that, TDI detected diastolic dysfunction more often than any other echocardiographic parameter. In our study, prevalence of diastolic dysfunction was $54.33 \%$. Poulsen et al. ${ }^{[19]}$ in their prospective observational study of 305 patients with type $2 \mathrm{DM}$ found that, abnormal LV filling is closely associated with abnormal myocardial perfusion on myocardial perfusion scintigraphy. Takeda et al. ${ }^{[20]}$ in their population of 544 consecutive Japanese DM patients with ejection fraction $\geq 50 \%$, found that diastolic dysfunction (impaired relaxation) plays a crucial role in the induction of HF with normal systolic function in DM patients, regardless of the severity of DM and renal dysfunction. These findings are partially comparable with our study where diastolic dysfunction was more prevalent with $\mathrm{HbA1c}>7.5$.

Poanta et al. ${ }^{[21]}$ in their study of 58 subjects found that, cardiac autonomic neuropathy was associated with LV diastolic dysfunction in patients with type $2 \mathrm{DM}$, but without clinical manifestation of the heart disease. Similarly Poirier et al. ${ }^{[22]}$ stated that, diastolic dysfunction and CAN (cardiac autonomic neuropathy) are associated in patients with otherwise uncomplicated well-controlled type 2 DM. Hameedullah et al. ${ }^{[23]}$ in their study population of 60 patients with type $2 \mathrm{DM}$ found that there was strong correlation between HbA1c level and diastolic indices $\left(' P{ }^{\prime}<0.05\right)$. Diastolic dysfunction was more frequent in poorly controlled diabetic patients, and its severity is correlated with glycaemic control. Similarly in our study, $\mathrm{HbA1c}>7.5 \%$ had higher prevalence of diastolic dysfunction compared to HbA1c $<7.5 \%$. C.M. Schannwell et al. ${ }^{[24]}$ in their study population of 87 subjects concluded that even young subjects with diabetes mellitus suffer from a diastolic dysfunction, while systolic ventricular function is normal. From the above discussion and comparison of present study findings with various studies, we found that there was high prevalence of diastolic dysfunction in subjects with asymptomatic type $2 \mathrm{DM}$, and it was correlated with age, duration of diabetes, HbA1c, dyslipidemia, autonomic neuropathy, retinopathy and various obesity indices. 


\section{Patil, et al.: Diastolic dysfunction in type 2 diabetes mellitus with normal LVEF}

Our study demonstrates that the incidence of pre-clinical diastolic dysfunction is high in type $2 \mathrm{DM}$ subjects. Furthermore, we found that there is a direct correlation between the duration of DM and diastolic dysfunction; and, that significant diastolic dysfunction occurs $>5$ years after the onset of DM independent of coronary disease or hypertension. Therefore, future studies should be conducted to test the hypothesis that screening and aggressive management of diabetic patients with pre-clinical diastolic dysfunction may delay the progression to heart failure.

\section{Study limitations}

The study was conducted on Indian general population. Thus, these findings need to be examined in different racial and ethnic groups. Homeostatic model assessment (HOMA) index for investigating fasting insulin concentration is not calculated in the present study due to resources limitations. HOMA index is considered as an independent factor for diastolic dysfunction.

\section{CONCLUSIONS}

Overall prevalence of diastolic dysfunction was $54.33 \%$ in asymptomatic type $2 \mathrm{DM}$ subjects in the present study. Asymptomatic type $2 \mathrm{DM}$ had significantly high prevalence of diastolic dysfunction as compared to healthy subjects. LV diastolic abnormalities were correlated with the duration of diabetes and with diabetic microangiopathies, like retinopathy and autonomic neuropathy. In the present study, $\mathrm{DM}$ was the strongest independent factor for $\mathrm{LV}$ diastolic dysfunction. This study confirms that asymptomatic diastolic dysfunction is more prevalent in subjects with type 2-DM. There was a significant correlation of LV diastolic dysfunction with the duration of diabetes, glycatedHbA1c levels, obesity indices (WC and WHR), retinopathy, autonomic neuropathy and hypertriglyceridemia, as determined by multivariate analysis. We conclude that early diagnosis and institution of treatment for diastolic dysfunction in the form of ACE inhibitors, angiotensin II receptor blockers, aldosterone antagonists, diuretics etc. depending on clinical scenario, will reduce the morbidity and improve the outcome of diastolic HF. In order to improve the current poor prognosis in subjects with DM, the treatment of diastolic HF must be optimised. Subjects with DM type 2 should be screened for sub clinical diastolic dysfunction by echocardiography.

\section{REFERENCES}

1. Kazik A, Wilczek K, Poloński L. Management of diastolic heart failure. Cardiol J 2010;17:558-65.
2. From AM, Scott CG, Chen HH. Changes in diastolic dysfunction in diabetes mellitus over time. Am J Cardiol 2009;103:1463-6.

3. Ommen SR, Nishimura RA, Appleton CP, Miller FA, Oh JK, Redfield MM, et al. Clinical utility of Doppler echocardiography and tissue Doppler imaging in the estimation of left ventricular filling pressures: A comparative simultaneous Doppler-catheterization study. Circulation 2000;102:1788-94.

4. OH JK, Seward JB, Tajik AJ. Assessment of diastolic dysfunction and diastolic heart failure. In: Oh JK, editor. The Echo Manual. $3^{\text {rd }}$ ed. New Dehli: Wolters Kluwer; 2006. p. 120-41.

5. Executive summary of third report of the cholesterol education program (NECP) Expert panel of detection, Evaluation, and treatment of high blood cholesterol In Adults. (Adult Treatment Panel III). JAMA 2001;285:2486-97.

6. Gupta R, Majumdar S. Correlation of waist-hip ratio with coronary heart disease and risk factor prevalence in a rural male population. Indian Heart J 1994;46:145-8.

7. Mishra TK, Rath PK, Mohanty NK, Mishra SK. Left ventricular systolic and diastolic dysfunction and their relationship with microvascular complications in normotensive, asymptomatic patients with type 2 diabetes mellitus. Indian Heart J 2008;60:548-53.

8. Soldatos G, Jandeleit-Dahm K, Thomson H, Formosa M, D’orsa K, Calkin AC, et al. Large artery biomechanics and diastolic dysfunctionin patients with Type 2 diabetes. Diabet Med 2011;28:54-60.

9. Sacre JW, Franjic B, Jellis CL, Jenkins C, Coombes JS, Marwick TH. Association of cardiac autonomic neuropathy with subclinical myocardial dysfunction in type 2 diabetes. JACC Cardiovasc Imaging 2010;3:1207-15.

10. van Heerebeek L, Hamdani N, Handoko ML, Falcao-Pires I, Musters RJ, Kupreishvili K, et al. Diastolic stiffness of the failing diabetic heart: Importance of fibrosis, advanced glycation end products, and myocyte resting tension. Circulation 2008;1;117:43-51.

11. Masugata H, Senda S, Goda F, Yoshihara Y, Yoshikawa K, Fujita N, et al. Left ventricular diastolic dysfunction in normotensive diabetic patients in various age strata. Diabetes Res Clin Pract 2008;79:91-6.

12. Annonu AK, Fattah AA, Mokhtar MS, Ghareeb S, Elhendy A. Left ventricular systolic and diastolic functional abnormalities in asymptomatic patients with non-insulin-dependent diabetes mellitus. J Am Soc Echocardiogr 2001;14:885-91.

13. Ashraf SM, Basir F. Association of hypertension and diastolic dysfunction with type-2 diabetes mellitus. Pak J Med Sci 2007;23:344-8.

14. Exiara T, Konstantis A, Papazoglou L, Kouroupi M, Kalpaka A, Mporgi $\mathrm{L}$, et al. Left ventricular diastolic dysfunction in diabetes mellitus Type 2. J Hypertens 2010;28:e294.

15. Diamant M, Lamb HJ, Groeneveld Y, Endert EL, Smit JW, Bax JJ, et al. Diastolic dysfunction is associated with altered myocardial metabolism inasymptomatic normotensive patients with well-controlled type 2 diabetes mellitus. J Am Coll Cardiol 2003;42:328-35.

16. Bonito PD, Cuomo S, Moio N, Sibilio G, Sabatini D, Quattrin S, et al. Diastolic dysfunction in patients with non-insulin-dependent diabetes mellitus of short duration. Diabet Med 1996;13:321-4.

17. From AM, Scott CG, Chen HH. The development of heart failure in patients with diabetes mellitus and pre-clinical diastolic dysfunction. J Am Coll Cardiol 2010;55:300-5.

18. Boyer JK, Thanigaraj S, Schechtman KB, Pérez JE. Prevalence of ventricular diastolic dysfunction in asymptomatic, normotensive patients with diabetes mellitus. Am J Cardiol 2004;93:870-875.

19. Poulsen MK, Henriksen JE, Dahl J, Johansen A, Gerke O, Vach W, et al. Left ventricular diastolic function in Type 2 diabetes mellitus: Prevalence and association with myocardial and vascular disease. Circ Cardiovasc Imaging 2010;3:24-31.

20. Takeda Y, Sakata Y, Mano T, Ohtani T, Kamimura D, Tamaki S, et al. Competing risks of heart failure with preserved ejection fraction in diabetic patients. Eur J Heart Fail 2011;13:664-9.

21. Poantă L, Fodor D, Albu A. Left ventricular function in patients with uncomplicated well-controlled diabetes mellitus. Med Ultrason 2010;12:184-7.

22. Poirier P, Bogaty P, Philippon F, Garneau C, Fortin C, Dumesnil JG. Preclinical diabetic cardiomyopathy: Relation of left ventricular diastolic dysfunction to cardiac autonomic neuropathy in men with uncomplicated well-controlled type 2 diabetes. Metabolism 2003;52:1056-61. 
Patil, et al:: Diastolic dysfunction in type 2 diabetes mellitus with normal LVEF

23. Hameedullah, Faheem M, Bahadar S, Hafizullah M, Najeeb S. Effect of glycaemic status on left ventricular diastolic function in normotensive type 2 diabetic patients. J Ayub Med Coll Abbottabad 2009;21:139-44.

24. Schannwell CM, Schneppenheim M, Perings S, Plehn G, Strauer BE. Strauer. Left ventricular diastolic dysfunction as an early manifestation of diabetic cardiomyopathy. Cardiology 2002;98:33-9.
How to cite this article: Patil VC, Patil HV, Shah KB, Vasani JD, Shetty P. Diastolic dysfunction in asymptomatic type 2 diabetes mellitus with normal systolic function. J Cardiovasc Dis Res 2011;2:213-22.

Source of Support: Nil, Conflict of Interest: None declared. 